\title{
Evaluation of Hexarelin Effects on Epileptic Seizures, Hippocampal Neuronal Damage and Memory Impairment after Pentylenetetrazole-Induced Acute Model in Rat
}

\author{
Yasar Tastemur ${ }^{1}$, Ekan Gumus ${ }^{2}$, Ahmet Sevki Taskıran ${ }^{3}$, Merve Ergul ${ }^{4}$, Bilal Sahin ${ }^{3}$, Ahmet Altun ${ }^{5}$ \\ ${ }^{1}$ Department of Anatomy, Sivas Cumhuriyet University School of Medicine, Sivas, Turkey
}

${ }^{2}$ Department of Histology and Embryology, Sivas Cumhuriyet University School of Medicine, Sivas, Turkey

${ }^{3}$ Department of Physiology, Sivas Cumhuriyet University School of Medicine, Sivas, Turkey

${ }^{4}$ Department of Pharmacology, Sivas Cumhuriyet University School of Pharmacy, Sivas, Turkey

${ }^{5}$ Department of Pharmacology, Sivas Cumhuriyet University School of Medicine, Sivas, Turkey

*Corresponding author: Ahmet Altun, Department of Pharmacology, Cumhuriyet University School of Medicine, 58140 Sivas, Turkey.

To Cite This Article: Ahmet Altun. Evaluation of Hexarelin Effects on Epileptic Seizures, Hippocampal Neuronal Damage and Memory Impairment after Pentylenetetrazole-Induced Acute Model in Rat. Am J Biomed Sci \& Res. 2019 - 5(4). AJBSR.MS.ID.000938. DOI: 10.34297/ AJBSR.2019.05.000938.

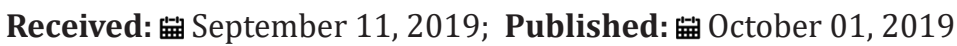

\begin{abstract}
Recent studies have demonstrated that ghrelin receptors have antiepileptic effects. The aim of this study was to investigate the effect of ghrelin receptor agonist hexarelin on pentylenetetrazole (PTZ)-induced seizures and post-seizure hippocampal damage. In our study, we used 42 male 230-250 g Wistar Albino rats. Animals were divided into seven groups as control, saline (PTZ; $1 \mathrm{ml} / \mathrm{kg}$ serum physiologic), positive control (5 $\mathrm{mg} / \mathrm{kg}$ diazepam), $50 \mu \mathrm{g} / \mathrm{kg}, 100 \mu \mathrm{g} / \mathrm{kg}, 200 \mu \mathrm{g} / \mathrm{kg}$ and $400 \mu \mathrm{g} / \mathrm{kg}$ hexarelin. $30 \mathrm{~min}$ after drugs administration at the indicated doses, PTZ was administered $45 \mathrm{mg} / \mathrm{kg}$ to induce an epileptic seizure. The animals were observed for $30 \mathrm{~min}$. Seizure stages (according to the Racine Scale) and first myoclonic jerk times (FMJ). 24 hours after PTZ injection, passive avoidance test was performed, and then brain tissues were removed. After the routine histological process, serial sections from brain tissues were stained with toluidine blue to determine neuronal damage. The hippocampal Cornu Ammonis CA1, CA3 and dentate gyrus regions were evaluated histopathologically. Statistical evaluation of the data was performed by oneway ANOVA, and multiple comparisons were determined by the Tukey test. Statistical significance was defined at $p<0.05$. Obtained data suggest that $200 \mu \mathrm{g} / \mathrm{kg}$ and $400 \mu \mathrm{g} / \mathrm{kg}$ hexarelin decreased seizure stages and increased FMJ compared to PTZ group (p<0,05). In addition, $200 \mu \mathrm{g} / \mathrm{kg}$ and 400 $\mu \mathrm{g} / \mathrm{kg}$ hexarelin improved retention time in passive avoidance compared to PTZ group ( $<<0,05)$. Furthermore, $200 \mu \mathrm{g} / \mathrm{kg}$ and $400 \mu \mathrm{g} / \mathrm{kg}$ hexarelin reduced neuronal damage in hippocampal CA1, CA3, and DG regions compared to PTZ group $(p<0,05)$. However, all these effects of hexarelin were not observed at $50 \mu \mathrm{g} / \mathrm{kg}$ and $100 \mu \mathrm{g} / \mathrm{kg}(\mathrm{p}>0,05)$. In conclusion, we suggest that hexarelin has protective effects on epileptic seizures and neuronal damage after PTZ dose-dependently.
\end{abstract}

Keywords: Hexarelin; Ghrelin; Epilepsy; Pentylenetetrazole; Passive avoidance; Neuronal damage

\section{Introduction}

Epilepsy is one of the most common chronic brain diseases affecting approximately $1 \%$ of the worldwide population. Even if epilepsy is a heterogeneous group of disorders, all kind of epilepsies shares the characteristic feature of recurrent unprovoked seizures, which can cause loss of awareness, injury, psychosocial disability and even mortality [1,2]. Anti-epileptic drugs (AEDs) provided via an oral route remain the mainstay of epilepsy treatment, though this method only achieves a satisfactory result with seizure control in approximately $70 \%$ of the patients. The remaining patients are unable to attain enough seizure alleviation. The phenomenon of unresponsiveness to oral administration seems to be multifactorial [3]. Whatever the reason is, epileptic patients need novel antiepileptic drugs which would have more potency and less toxicity [4]. It is not surprising that neuropeptides take attention in this seek. Neuropeptides were reported to be effective at the receptor level balancing the excitatory and inhibitory systems by regulating the release of GABA and glutamate neurotransmitters [5]. Ghrelin, an endogenous ligand of the growth hormone (GH) 
secretagogue receptor 1a (GHSR1a) [6] is predominantly produced by the stomach from its precursor proghrelin, which is synthesized by A-like cells [7]. After secretion, ghrelin is rapidly converted into the previously considered inactive form des-acyl-ghrelin [6] so that des-acyl-ghrelin represents the main circulating peptide of the ghrelin family of hormones [8]. Ghrelin and des-acyl-ghrelin have been involved in the regulation of food intake, locomotion, and energy balance [9].

Some studies have reported a relationship between epilepsy and ghrelin neuropeptide [10]. Saliva and serum ghrelin levels are reportedly reduced in epileptic patients [11]. Ghrelin levels were investigated only in acute PTZ injections [10]. In addition, it was suggested that low ghrelin and elevated nesfatin-1 levels might contribute to epilepsy pathophysiology [11]. Hexarelin is an analog of ghrelin that shows cardioprotective effects both in vivo and in vitro (via GHS-R1a) [12,13]. Hexarelin is chemically more stable and functionally more potent when compared with ghrelin [14], which makes hexarelin a promising substitute for ghrelin in the clinical applications. Although the relationship between epilepsy and grelin is known for a while, and it is also well-documented that hexarelin is a potent and reliable agent which mimics ghrelin's biological functions, as far as know, there are only a few studies about the effect of hexarelin on epilepsy and there is none focusing on neuroprotection in PTZ-induced epilepsy model. Considering this evidence, we aimed to investigate antiepileptic and neuroprotective effects of hexarelin in PTZ-induced epilepsy model.

\section{Materials and Methods}

\section{Animals}

All research designs were put into action following the guidelines of the Sivas Cumhuriyet University ethics committee. All animals used in this study were placed 12 hours of light / 12 hours of darkness in a cage and a temperature of $21-22^{\circ} \mathrm{C}$. Rats have free access to food and water

\section{Agent}

Hexarelin is dissolved in $0.9 \% \mathrm{NaCl}$, and pentylenetetrazol (PTZ) is dissolved in physiological serum (SF). Agents and chemicals were purchased from "Sigma Aldrich Co., St. Louis, MO, USA". All holds are prepared just before the experiments.

\section{Design}

42 (220-250 g) "Wistar Albino" male rats were used for our study.

\section{Results}

\section{The Antiepileptic Effect of Hexarelin}

Table 1: Effect of Hexarelin on seizures threshold (latency) in PTZ-induced seizures in mice.

\begin{tabular}{|c|c|c|}
\hline Group & Racine Scale & Latency to the 1st \\
\hline myoclonic seizures (sec) & & None \\
\hline Control & None & N \\
\hline
\end{tabular}

Seven study groups are designed as follows;

a. 1.Control group,

b. 2. The saline group (PTZ; $1 \mathrm{ml} / \mathrm{kg}$ in SF),

c. Positive control (2.5 mg / kg diazepam),

d. $\quad 50 \mu \mathrm{g} / \mathrm{kg}$ hexarelin,

e. $\quad 100 \mu \mathrm{g} / \mathrm{kg}$ hexarelin,

f. $\quad 200 \mu \mathrm{g} / \mathrm{kg}$ hexarelin,

g. $\quad 400 \mu \mathrm{g} / \mathrm{kg}$ hexarelin.

Thirty minutes after drug administration, PTZ was given45 $\mathrm{mg} / \mathrm{kg}$ to induce epileptic seizures. The animals were observed for 30 minutes. The seizure stage (according to the Racine Scale) and the first myoclonic jerk times (FMJ). Twenty-four hours after PTZ injection, a passive avoidance test was carried out, and then brain tissue was extracted for biochemical and histopathological examination. After a routine histological process, the serial part of the brain tissue is stained with blue toluidine to determine nerve damage. The hippocampal area "Cornu Ammonis (CA)" CA-1, CA3 , and "dentate gyrus (DG)" was evaluated histopathologically to evaluate neuronal damage.

\section{Histopathology}

The part of the brain that is fixed in a formaldehyde solution is stained with toluidine blue to identify the number of dark neurons. All parts of the network were examined and photographed with a digital camera "Olympus C-5050" in the microscope "Olympus BX51". In the CA-1, CA-2 and DG hippocampal regions, dark neurons and survival neurons were counted in six parts for each animal studied ( $\mathrm{n}=3$ for each group) with an image analysis system ("Image-Pro Express 1.4.5, Media Cybernetics, Inc. USA"). The number of dark neurons is given as a percentage (blue-stained toluidine neurons * 100 / survival neurons). The inspection was carried out blindly.

\section{Statistic analysis}

Data generated by this study is stated as "mean \pm standard error of the mean (SEM)". Data were analyzed using "SPSS Version 21.0". RCS scores, FMJ times, and the number of dark neurons were evaluated using one-way analysis of variance (ANOVA). The Tukey test has been chosen as a posthoc test. $\mathrm{P}$ values were statistically significant when $\mathrm{p}<0.05$. 


\begin{tabular}{|c|c|c|}
\hline Saline (PTZ 45 mg/kg, i.p.) & $4,50 \pm 0,22$ & $57,50 \pm 0,22$ \\
\hline Positive Control (Diazepam 5 mg/kg, i.p.) & $1,00 \pm 0,3^{*}$ & None \\
\hline Hexarelin (50 $\mu \mathrm{g} / \mathrm{kg}$, i.p.) & $4,50 \pm 0,22$ & $59,16 \pm 2,91$ \\
\hline Hexarelin (100 $\mu$ g /kg, i.p.) & $4,33 \pm 0,33$ & $66,00 \pm 5,14$ \\
\hline Hexarelin (200 $\mu \mathrm{g} / \mathrm{kg}$, i.p.) & $3,5 \pm 0,22^{*}$ & $75,33 \pm 4,57^{*}$ \\
\hline Hexarelin $(400 \mu \mathrm{g} / \mathrm{kg}$, i.p. $)$ & $2,83 \pm 0,3^{*}$ & $92,00 \pm 3,37^{*}$ \\
\hline
\end{tabular}

The PTZ-induced epilepsy mouse model has been used to test whether hexarelin has an antiepileptic effect in rats. Table 1 shows the Racine Scale Score and latency to the first myoclonic seizures in the two control groups, saline, positive control, and hexarelin $50,100,200$, and $400 \mathrm{mg} / \mathrm{kg}$. While diazepam reduced the Racine Scale Score and latency to myoclonic seizures significantly $(1.00 \pm$ 0.36 vs $4.50 \pm 0.22$ and 57.500 .22 vs none respectively) ( $<<0.05$ ), on the other hand, low-dose hexarelin (50 and 100 Hexarelin $\mu \mathrm{g}$ / $\mathrm{kg}$ ) doses create a statistically significant effect on both the Racine Scale and latency indicating no Hexarelin antiepileptic effect in the PTZ-induced epilepsy model at low doses ( $p>0,05)$. However, higher hexarelin doses (200 and $400 \mathrm{ug} / \mathrm{kg}$ ) succeeded in creating a statistically significant antiepileptic effect when compared to the PTZ group (for Racine Scale Scores of $3.5 \pm 0.22$ vs $4.50 \pm 0.22$ and $2,83 \pm 0.30$, respectively (for myoclonic latency seizures to 1 , $75.33 \pm 4.57$ vs $57.50 \pm 0.22$ and $92.00 \pm 3.37$ vs. $57.50 \pm 0.22$, respectively) ( $\mathrm{p}<0.05)$. Although 200 and $400 \mathrm{ug} / \mathrm{kg}$ create strong antiepileptic effects, none of the doses are strong enough to achieve the antiepileptic effect of positive control (Diazepam 5mg / kg) (Table 1).

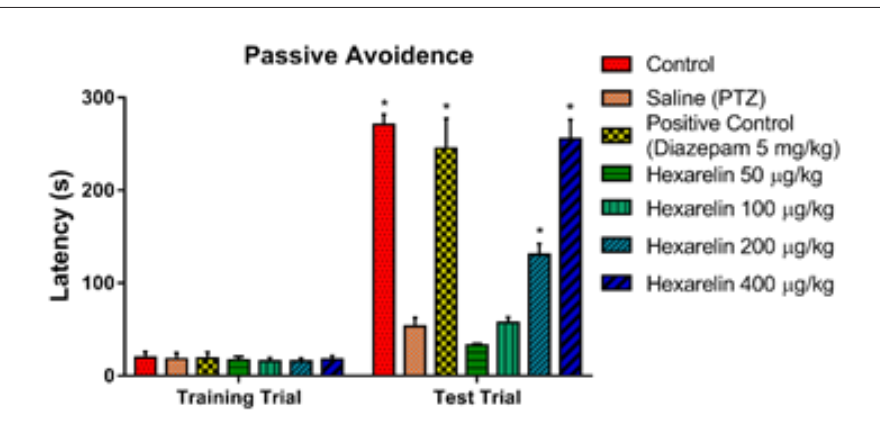

Figure 1: Passive Avoidance Latencies in both Training and Test Trial (s).

Figure 1 shows passive avoidance latencies in both training and test trial. After a successful training trial period, as shown in the control group, animals successfully learned to avoid the harmful stimulus which has been proven by increased latency durations. Nevertheless, passive avoidance latencies dropped dramatically in animals with PTZ-induced seizures $(\mathrm{p}<0.05)$. In the presence of hexarelin, latencies were recovered and went back to control levels in a dose-dependent manner where 50 and $100 \mu \mathrm{g} / \mathrm{kg}$ hexarelin did not have any effect on passive avoidance latencies ( $p>0.05), 200$ and $400 \mu \mathrm{g} / \mathrm{kg}$ hexarelin restored latencies significantly $(\mathrm{p}<0.05)$. There was no significant difference between $400 \mu \mathrm{g} / \mathrm{kg}$ hexarelin and the control group ( $p>0.05$ ) (Figure 1).

\section{Evaluation of Dark Neurons}
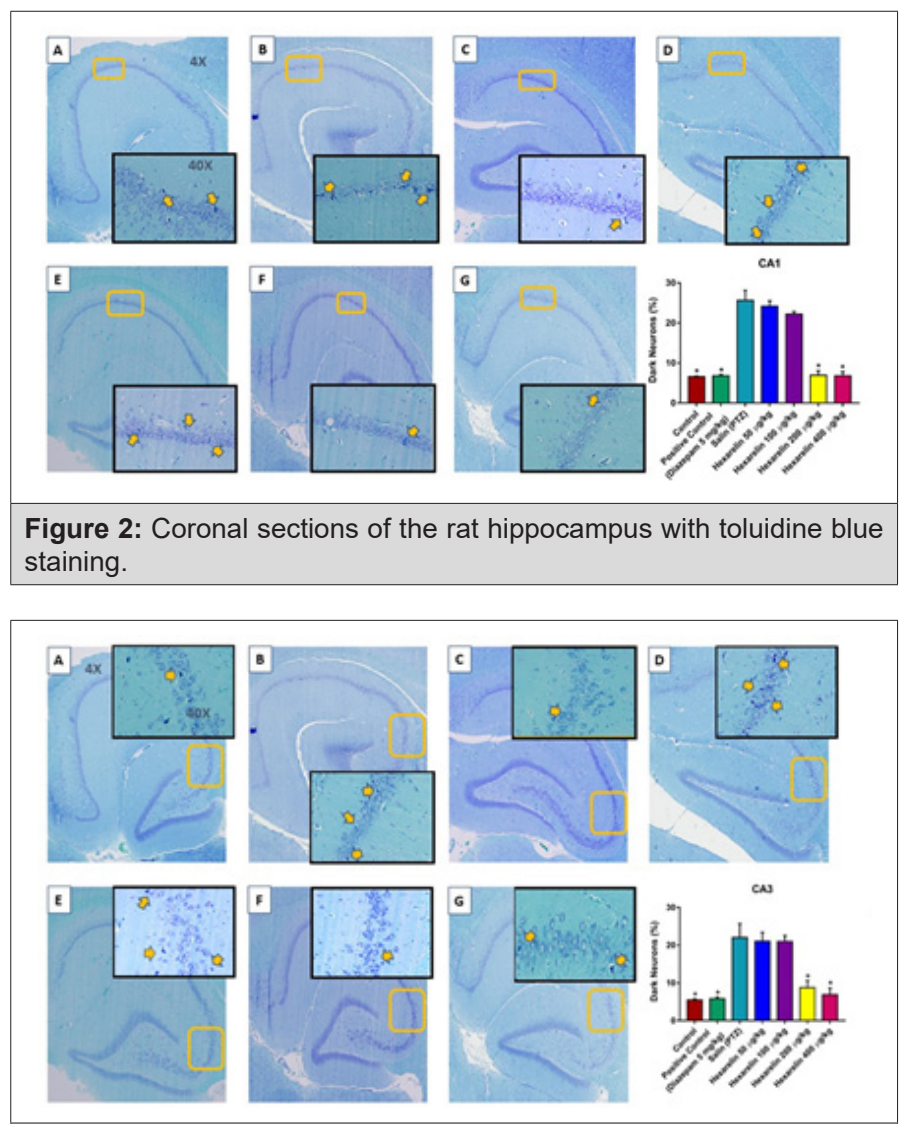

Figure 3: Coronal sections of the rat hippocampus with toluidine blue staining.

Dark neurons are recognized by neuronal shrinkage, cytoplasmic eosinophilia, nuclear pyknosis, and surrounding spongiosis in total hippocampal formation (Figure 2A, 2E, 2I) and CA1 (Figure 2), CA3 (Figure 3) and DG (Figure 4) hippocampal formation. The administration of hexarelin at doses of 200 and $400 \mu \mathrm{g} / \mathrm{kg}$ significantly prevented the production of dark neurons due to PTZ-induced seizures in the CA1, CA3 and DG regions of the hippocampus ( $\mathrm{p}<0.05$ ) (Figure 5A-5C). However, there were no significant differences between the doses of 50 and $100 \mu \mathrm{g} / \mathrm{kg}$ of the Hexarelin and PTZ groups in the CA1, CA3 and DG hippocampus regions at dark neuron points ( $\mathrm{p}>0.05$ ) (Figure 5A-5C). In addition, there was no significant difference between $200 \mu \mathrm{g} / \mathrm{kg}$ and 400 $\mu \mathrm{g} / \mathrm{kg}$ hexarelin in CA1, CA3 and DG hippocampus regions at dark neuron points ( $p>0.05$ ) (Figure 2). Basophilic (dark) neurons (yellow arrow) distributed between normal pyramidal neurons in the hippocampal CA1 (Cornu Ammonis) region. (A) Control, (B) PTZ, (C) positive control, (D) $50 \mu \mathrm{g} / \mathrm{kg}$ Hexarelin, (E) $100 \mu \mathrm{g} /$ 
kg Hexarelin, (F) $200 \mu \mathrm{g} / \mathrm{kg}$ Hexarelin, (G) $400 \mu \mathrm{g} / \mathrm{kg}$ Hexarelin groups. Comparison of dark neuron numbers for each area in CA1 (F) areas between groups. Data are presented as mean \pm SEM. *P $<0.05$ in comparison with the control group (Figure 3).
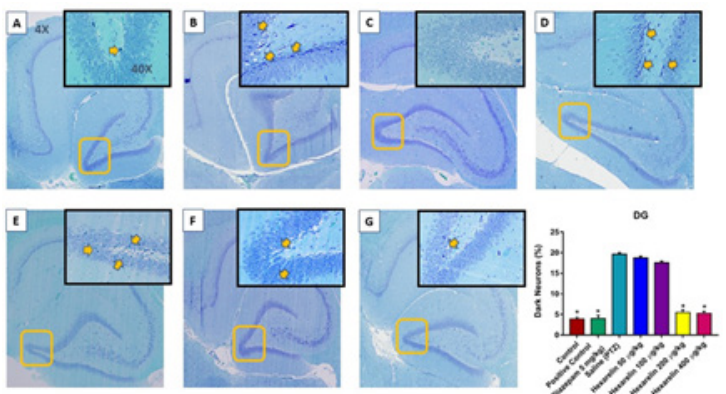

Figure 4: Coronal sections of the rat hippocampus with toluidine blue staining.
Basophilic (dark) neurons (yellow arrow) distributed between normal pyramidal neurons in the hippocampal CA3 (Cornu Ammonis) region. (A) Control, (B) PTZ, (C) positive control, (D) $50 \mu \mathrm{g} / \mathrm{kg}$ Hexarelin, (E) $100 \mu \mathrm{g} / \mathrm{kg}$ Hexarelin, (F) $200 \mu \mathrm{g} / \mathrm{kg}$ Hexarelin, (G) $400 \mu \mathrm{g} / \mathrm{kg}$ Hexarelin groups. Comparison of dark neuron numbers for each area in CA1 (F) areas between groups. Data are presented as mean \pm SEM. ${ }^{*} \mathrm{P}<0.05$ in comparison with the control group (Figure 4). Basophilic (dark) neurons (yellow arrow) distributed between normal pyramidal neurons in the hippocampal DG (dentate gyrus) region. (A) Control, (B) PTZ, (C) positive control, (D) $50 \mu \mathrm{g} / \mathrm{kg}$ Hexarelin, (E) $100 \mu \mathrm{g} / \mathrm{kg}$ Hexarelin, (F) $200 \mu \mathrm{g} / / \mathrm{kg}$ Hexarelin, (G) $400 \mu \mathrm{g} / / \mathrm{kg}$ Hexarelin groups (Figure 5). Dark Neuron percentage at A) CA1, B) CA3 and C) DG regions in control, PTZ, Diazepam, Hexarelin 50, Hexarelin 100, Hexarelin 200 and Hexarelin $400 \mu \mathrm{g} / \mathrm{kg}$ groups.

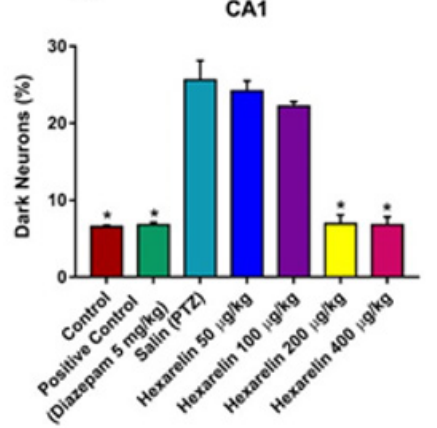

B

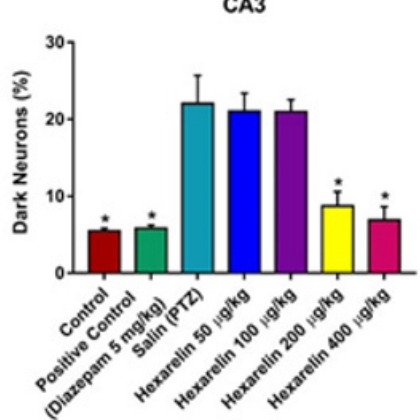

C

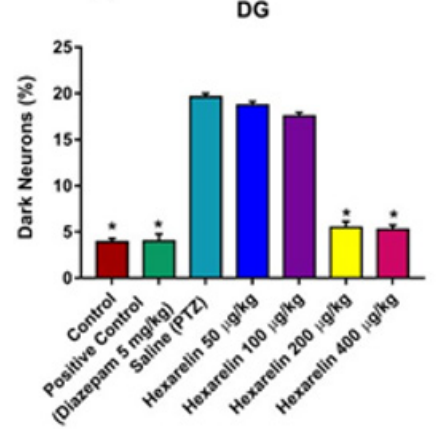

Figure 5: Dark Neuron percentage at A) CA1, B) CA3 and C) DG regions in control.

\section{Discussion}

The seizures are occurred because of abnormal hypersynchronous paroxysmal cerebral discharges from the neurons, which eventually results in irreversible damage to them and their surroundings. There is tremendous development in epilepsy treatment in the last decades. Nevertheless, most of the treatment agents are focused on ion channels to prevent seizures. Yet, they are not able to prevent the neural damage which comes after the seizures anyway. That is why an ideal antiepileptic agent should able to both prevent the seizure and provide neuroprotective effect if the seizure happens anyway. It is also essential to preserve residual neural capacity even if a seizure does not occur again [15]. In the current study, we have investigated the antiepileptic effects of hexarelin, along with its impact on memory and nerve protection. Ghrelin is a multifunctional 28-amino acid hormone which is known as the endogenous ligand of the synthetic growth hormone secretagogue receptor 1a (GHS-R) ligands [16]. It is not a secret that there is a link between ghrelin and abnormal electric activity in the brain. It is even called anticonvulsant neuropeptide [17]. It has been shown that both blood and brain levels of ghrelin decrease in epilepsy. Ataie et al. [10] suggested that reduction of plasma acylated ghrelin after PTZ-induced seizures could be attributed to its uptake by the brain to represent an antiepileptic effect [10]. In the present study, we managed to show that hexarelin has an inhibitory effect on the PTZ-induced seizures in rats. There are few studies in the literature, including Obay et al. [18] which shows that exogenous ghrelin administration may attenuate or inhibit the seizures in the PTZ-induced rats. It seems the antiepileptic effect of ghrelin analogs is related to the method how the seizures induced, since Biagini et al. [19] could not show strong antiepileptic effect of hexarelin in status epilepticus model, and Han et al. [20] could demonstrate a strong antiepileptic effect of ghrelin in pilocarpineinduced inflammatory epilepsy model. When the whole literature investigated globally, it is seen that our promising results about the antiepileptic effect of hexarelin are supported by the literature.

There is a very limited amount of information about the effect of hexarelin on cognitive functions. Evidence obtained in an animal model conducted by Ghersi et al. [21] suggests that ghrelin can enhance long-term potentiation and, thus, memory. Another study investigated the effects of growth hormone-releasing hormone on cognitive function in adults with cognitive impairments and achieved positive results [22]. It is not about hexarelin. In the present study, we showed that especially high doses of hexarelin preserved the cognitive function when compared to non-treated 
PTZ-induced epilepsy group. There is again a minimal number of studies and speculations that Growth Hormone Secretagogues have neuroprotective effects [23], but there is none, as far as we know, which proves it via histological sections. In our study, we aimed to evaluate if there is a neuroprotective effect of hexarelin by determining the numbers of dark neurons which were directly related to the neuronal damage. Our results show that $400 \mu \mathrm{g} / \mathrm{kg}$ Hexarelin reduced the number of dark neurons to the control levels, which indicates the strong neuroprotective effect at the attributed doses. This phoneme is in accordance with the literature results. Although literature knowledge is very limited in this matter, when more in-depth literature search is done, the protective effects of ghrelin analogs are not limited to the neurons. Cytoprotective abilities of ghrelin analogs have been revealed in cardiac, neuronal, gastrointestinal, and hepatic cells, representing a comprehensive spectrum of protection of parenchymal organs [24].

Finally, we believe that preventing seizures, protecting memory, and neurons by hexarelin are significant because of one other beneficial feature of the ghrelin analogs. The literature suggests that ghrelin levels are higher in patients with good response to antiepileptic drugs [25]. This extra feature may open the possibility of combination antiepileptic drugs with hexarelin.

\section{Conclusion}

In conclusion, with the strong antiepileptic, cognition, and neuroprotective effects, hexarelin may be a promising candidate agent for epilepsy treatment and can be administrated in combination with the conventional antiepileptic drugs.

\section{Reference}

1. Goldberg EM, Coulter DA (2013) Mechanisms of epileptogenesis: A convergence on neural circuit dysfunction. Nat Rev Neurosci 14(5): 337349.

2. Shorvon SD (2009) Drug treatment of epilepsy in the century of the ILAE: The second 50 years, 1959-2009. Epilepsia 50(3): 93-130.

3. Oby E, Janigro D (2006) The blood-brain barrier and epilepsy. Epilepsia 47(11): 1761-1774.

4. Pitkänen A, Lukasiuk K (2009) Molecular and cellular basis of epileptogenesis in symptomatic epilepsy. Epilepsy Behav 14(1): 16-25.

5. Clynen E, Swijsen A, Raijmakers M, Hoogland G, Rigo JM (2014) Neuropeptides as Targets for the Development of Anticonvulsant Drugs. Mol Neurobiol 50(2): 626-646.

6. Kojima M (2005) Ghrelin: Structure and Function. Physiol Rev 85(2): 495:522.

7. Che, CY, Asakawa A, Fujimiya M, Lee S, Inui A (2009) Ghrelin Gene Products and the Regulation of Food Intake and Gut Motility. Pharmacol Rev 61(4): 430-481.

8. Veldhuis JD, Bowers CY (2010) Integrating GHS into the Ghrelin System. Int J Pept Pp. 879503.
9. Asakawa A, Inui A, Fujimiya M, Sakamaki R, Shinfuku N, et al. (2005) Stomach regulates energy balance via acylated ghrelin and desacyl ghrelin. Gut 54(1): 18-24.

10. Ataie Z, Golzar MG, Babri S, Ebrahimi H, Mohaddes G (2011) Does ghrelin level change after epileptic seizure in rats? Seizure 20(4): 347-349.

11. Aydin S, Dag E, Ozkan Y, Erman F, Dagli AF, et al. (2009) Nesfatin-1 and ghrelin levels in serum and saliva of epileptic patients: Hormonal changes can have a major effect on seizure disorders. Mol Cell Biochem 328(12): 49-56.

12. Ma Y, Zhang L, Launikonis BS, Chen C (2012) Growth hormone secretagogues preserve the electrophysiological properties of mouse cardiomyocytes isolated from in Vitro ischemia/reperfusion heart. Endocrinology 153(11): 5480-5490.

13. Ma Y, Zhang L, Edwards JN, Launikonis BS, Chen C (2012) Growth hormone secretagogues protect mouse cardiomyocytes from in vitro ischemia/reperfusion injury through regulation of intracellular calcium. PLoS One 7(4): e35265.

14. Mao Y, Tokudome T, Kishimoto I (2014) The cardiovascular action of hexarelin. J Geriatr Cardiol 11(3): 253-258.

15. Acharya MM, Hattiangady B, Shetty AK (2008) Progress in neuroprotective strategies for preventing epilepsy. Prog Neurobiol 84(4): 363-404.

16. Lim CT, Kola B, Korbonits M (2011) The ghrelin/GOAT/GHS-R system and energy metabolism. Rev Endocr Metab Disord 12(3): 173-186.

17. Portelli J, Michotte Y, Smolders I (2012) Ghrelin: An emerging new anticonvulsant neuropeptide. Epilepsia 53(4): 585-585.

18. Obay BD, Tasdemir E, Tümer C, Bilgin HM, Sermet A (2007) Antiepileptic effects of ghrelin on pentylenetetrazole-induced seizures in rats. Peptides 28(6): 1214-1219.

19. Biagini G, Torsello A, Marinelli C, Gualtieri F, Vezzali R, et al. (2011) Beneficial effects of desacyl-ghrelin, hexarelin and EP-80317 in models of status epilepticus. Eur J Pharmacol 670(1): 130-136.

20. Han K, Wang QY, Wang CX, Luan SY, Tian WP, et al. (2018) Ghrelin improves pilocarpine-induced cerebral cortex inflammation in epileptic rats by inhibiting NF-kB and TNF- $\alpha$. Mol Med Rep 18(4): 3563-3568.

21. Ghersi MS, Gabach LA, Buteler F, Vilcaes AA, Schiöth HB, et al. (2015). Ghrelin increases memory consolidation through hippocampal mechanisms dependent on glutamate release and NR2B-subunits of the NMDA receptor. Psychopharmacology (Berl) 232(10): 1843-1857.

22. Baker LD, Barsness SM, Borson S, Merriam GR, Friedman SD, et al. (2012). Effects of growth hormone-releasing hormone on cognitive function in adults with mild cognitive impairment and healthy older adults: Results of a controlled trial. Arch Neurol 69(11): 1420-1429.

23. Johansson I, Destefanis S, Åberg ND, Åberg MAI, Blomgren K, et al. (2008). Proliferative and protective effects of growth hormone secretagogues on adult rat hippocampal progenitor cells. Endocrinology 149(5): 2191-2199.

24. Berlanga Acosta J, Abreu Cruz A, García del Barco Herrera D, Mendoza Marí Y, Rodríguez Ulloa A, et al. (2017) Synthetic growth hormone-releasing peptides (GHRPS): A historical appraisal of the evidences supporting their cytoprotective effects. Clin Med Insights Cardiol 11: 1179546817694558

25. Marchiò M, Roli L, Giordano C, Caramaschi E, Guerra A, et al. (2018) High plasma levels of ghrelin and des-acyl ghrelin in responders to antiepileptic drugs. Neurology 91(1): e62-e66. 